\title{
Non Unuiform Phased array Beamforming with Covariance Based Method
}

\author{
Amirsadegh Roshanzamir ${ }^{1}$, M. H. Bastani ${ }^{2}$ \\ ${ }^{1,2}$ (Sharif university of technology)
}

\begin{abstract}
Many papers have investigated phased array radars beam-forming so far. In these papers the power at a specific point of interest is written by a combination of transmitted power by means of each antenna. In this paper we will introduce a new approach which will use of cross correlation matric like MIMO radars to define beam-pattern of array and then by introducing an optimization problem to steering the available power at desire directions, it is shown that with an approach to optimum choosing of antenna placement, it is possible to concentrate the transmitter power on desire direction better than conventional phased array antennas with half wave length spacing. It should be say that some papers have investigated non uniform arrays but those papers usually use of multiple of half wave length in their elements spacing and in addition their approach to choosing these spaces is different and pretty more complicated from presented approach. Simulation is performed to analyze the presented algorithm and compare it's performance with conventional phased arrays with half wave length spacing for linear and planar array. MATLAB software is being employed for simulations.
\end{abstract}

Keywords — beamforming, covariance based methods, MIMO radars, phased array

\section{INTRODUCTION}

Arrays are today used for many applications and the view and terminology is quite different. The principle of phased arrays is to generate a plane wavefront from a large number of elementary spherical waves. These spherical waves are approximately realized by elementary antenna elements with near omnidirectional characteristic. The application of suitable excitations at the elementary antennas and the summation of all signals on receive is called beamforming.

The main advantage of phased array antennas is the nearly infinitely fast switching of the look direction of the array. This allows to illuminate the search space according to some optimality criterion instead of according to a continuous mechanical movement. All aspects of optimizing target illumination the received energy are denoted by the key word energy management, which is the principle advantage of phased arrays. Particular components of energy management are [1-7]:

- Performance of different radar task in time multiplex, like search and tracking of multiple targets. This allows to use one phased array radar as multi-function radar.

- Optimization of individual radar task: optimized waveforms for search, acquisition and track, high precision measurements when required, variable beam shapes, optimized algorithms for tracking (the radar is "steered" by the tracking algorithm and a priori information)

- Lower prime energy consumption (only for active array, saving of about a factor of 2)

- High mean time between failure (MTBF) due to graceful degradation (only active array)

- If spatial samples over the antenna aperture are available: adaptive beam forming (ABF), space-time adaptive processing (STAP) super-resolution

In this paper we will introduce a new approach which will use of cross correlation matric like MIMO radars to define beam-pattern of array and then by introducing an optimization problem to steering the available power at desire directions, it is shown that with an approach to optimum choosing of antenna placement, it is possible to concentrate the transmitter power on desire direction better than conventional phased array antennas with half wave length spacing. It should be say that some papers have investigated non uniform arrays but those papers usually use of multiple of half wave length in their elements spacing and in addition their approach to choosing these spaces is different and pretty more complicated from presented approach. Simulation is performed to analyze the presented algorithm and compare it's performance with conventional phased arrays with half wave length spacing for linear and planar arrays.

The present study has five sections as follows; section I presents a brief introduction. Utilization of cross-correlation matric and covariance based method formulation for desire problem is discussed in section II. Our main goal and algorithm is provided in section III. Numerical examples and simulation results are discussed in section IV. Section V is focused on conclusion and references are presented at the end of the paper. 


\section{COVAVIANCE BASED METHOD FORMULATION}

Assume that we have a collection of $\mathrm{N}$ transmitter antennas which are located at known coordinates $\mathrm{x}_{\mathrm{i}}=\left(\mathrm{x}_{1, \mathrm{i}}, \mathrm{x}_{2, \mathrm{i}}, \mathrm{x}_{3 \mathrm{i}}\right)=(\mathrm{x}, \mathrm{y}, \mathrm{z})$ in some spherical coordinate along the $\mathrm{z}$-axis as shown in Fig. 1. In the presented study and in all of the examples and formulas of current paper, it is assumed that these transmitter antennas are along the z-axis.

It is assume that each transmitter antenna is driven by a specific signal on the carrier frequency of $\mathrm{f}_{\mathrm{c}}$ or with wavelength of $\lambda$ and complex envelope of $s_{i}(t), i=1, \ldots, N$. At a specific point in space with distance of $r$ and direction of $\mathrm{k}(\theta, \phi)$ from the transmitter antenna, each radiated signal $\mathrm{s}_{\mathrm{i}}(\mathrm{t})$ gives rise to a "signal" the far field at radius $r$, with complex envelope given by

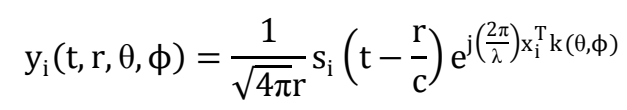

Where, in this equation, $\mathrm{k}$ is a unit vector in the $(\theta, \phi)$ direction.

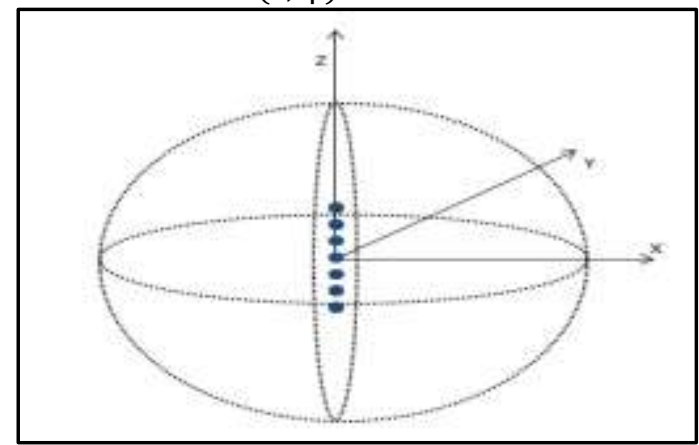

Fig. 1. T/R modules and spherical coordinate system

At the far field, these signals add linearly and the radiated powers $P_{i}$ add linearly as well. At this point assume that the $\mathrm{i}$-th element location is on the $\mathrm{z}$-axis at coordinate $\mathrm{z}_{\mathrm{i}}$. The signal at position $(r, \theta, \phi)$ resulting from all of the transmitted signals at far field will be:

$$
\begin{aligned}
& y(t, r, \theta, \phi)=\sum_{i=1}^{N} y_{i}(t, r, \theta, \phi) \\
= & \frac{1}{\sqrt{4 \pi}} \sum_{\mathrm{i}=1}^{\mathrm{N}} \mathrm{s}_{\mathrm{i}}\left(\mathrm{t}-\frac{\mathrm{r}}{\mathrm{c}}\right) \mathrm{e}^{\mathrm{j}\left(\frac{2 \pi z_{\mathrm{i}}}{\lambda}\right) \sin (\theta)}
\end{aligned}
$$

The power density of the entire signals then given by

$$
=\frac{1}{4 \pi \mathrm{r}^{2}} \sum_{\mathrm{k}=1}^{\mathrm{N}} \sum_{\mathrm{l}=1}^{\mathrm{N}}<\mathrm{s}_{\mathrm{k}}(\mathrm{t}) \mathrm{s}_{\mathrm{l}}^{*}(\mathrm{t})>\mathrm{e}^{\mathrm{j}\left(\frac{2 \pi\left(\mathrm{z}_{\mathrm{k}}-\mathrm{z}_{1}\right)}{\lambda}\right) \sin (\theta)}
$$

And it is known that the complex signal cross-correlation is defined by

$$
\mathrm{R}_{\mathrm{kl}}=<\mathrm{s}_{\mathrm{k}}(\mathrm{t}) \mathrm{s}_{\mathrm{l}}^{*}(\mathrm{t})>
$$

With defining the direction vector as below

The normalized power density $\mathrm{P}(\theta, \phi)$ of signals, in (W/ster), would be:

$$
\mathrm{a}(\theta)=\left[\mathrm{e}^{\mathrm{j}\left(\frac{2 \pi z_{1}}{\lambda}\right) \sin (\theta)}, \ldots, \mathrm{e}^{\mathrm{j}\left(\frac{2 \pi z_{\mathrm{N}}}{\lambda}\right) \sin (\theta)}\right]^{\mathrm{T}}
$$

$$
P(\theta, \phi)=\frac{1}{4 \pi} \sum_{k=1}^{N} \sum_{l=1}^{N} R_{k l} e^{\frac{j 2 \pi}{\lambda}\left(z_{k}-z_{l}\right) \sin (\theta)}
$$


Recognizing that (6) is quadratic form in the Hermitian matrix R which is the cross-correlation matrix of signals, this can be written compactly as

$$
\mathrm{P}(\theta, \phi)=\frac{1}{4 \pi} \mathrm{a}^{*}(\theta) \operatorname{Ra}(\theta)
$$

This normalized power density $\mathrm{P}(\theta, \phi)$ is exactly the beampattern which is desirable to find $[8,9]$.

In the following some examples of beampatterns produce from such a cross-correlation matrix has been shown. Fig. 2 shows the beampattern produced by signal cross-correlation matrix of (8), (9) and (10) respectively. It is noticeable that these figures are beam-patterns of 10-element uniform linear array (ULA) with half-wavelength spacing.

$$
\left[\begin{array}{ccc}
1 & \cdots & 1 \\
\vdots & \ddots & \vdots \\
1 & \cdots & 1
\end{array}\right]
$$

$$
\left[\begin{array}{ccc}
0.8^{0} & \cdots & 0.8^{9} \\
\vdots & \ddots & \vdots \\
0.8^{9} & \cdots & 0.8^{0}
\end{array}\right]
$$

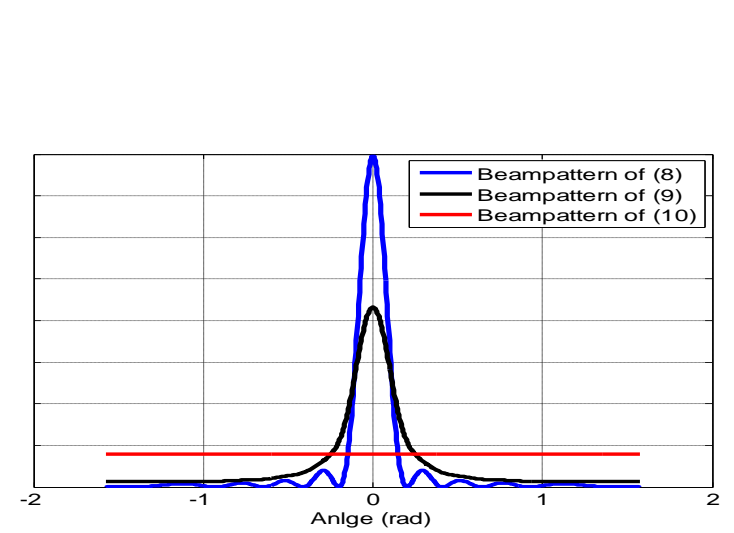

Fig. 2. Beampattern respect to (7). The blue one is corresponds to cross-correlation matrix of (8), The black one is corresponds to cross-correlation matrix of (9) and the red one is corresponds to cross-correlation matrix of

In general case the elements of the signal cross-correlation matrix are complex values except the diagonal elements that are real. This general case is related to MIMO radars but in the case of phased array radar, all the transmitter signals are correlated with each other and then absolute value of all the elements in $R$, are equal to 1(blue one at Fig. 2).

\section{USING COVARIANCE MATRIX TO OPTIMIZE BEAMPATTERN}

In this section we introduce our cost function. Let us assume that there are $\mathrm{K}\left(\mathrm{K}<\mathrm{N}_{\mathrm{t}}\right)$ targets of interest which $N_{t}$ is total number of transmitter elements. Without loss of generality, they are assume at locations $\left\{\theta_{\mathrm{k}}\right\}_{\mathrm{k}=1}^{\mathrm{K}}$. Then the cumulated power of the probing signals at the target locations is given by

Where

$$
\sum_{\mathrm{k}=1}^{\mathrm{K}} \mathrm{a}^{*}\left(\theta_{\mathrm{k}}\right) \operatorname{Ra}\left(\theta_{\mathrm{k}}\right) \triangleq \operatorname{tr}(\mathrm{RB})
$$

$$
\boldsymbol{B}=\sum_{k=1}^{K} \boldsymbol{a}\left(\theta_{k}\right) \boldsymbol{a}^{*}\left(\theta_{k}\right)
$$

In [10] authors have defined a cost function of $\max _{\mathrm{R}} \operatorname{tr}(\mathrm{R} \widehat{\mathrm{B}})$ with some constraints on correlation matric of R. In this equation $\widehat{B}$ is an estimate of $B$ which means that some prior information is available on target locations. In our case as we are working on phased array radars, all the elements of cross correlation matric are equal to 1 , then we cannot solve that problem respect to R. As a result we will form this equation respect to a vector, and consequently we use of direct estimation of $\theta$ or a, instead of $B$. Then our cost function will be the form below 


$$
\begin{gathered}
\max _{\mathrm{a}} \operatorname{tr}(\mathrm{R} \widehat{\mathrm{B}}) \\
\boldsymbol{R}=\text { allonematric } \\
\widehat{\mathrm{B}}=\sum_{\begin{array}{c}
\mathrm{k}=1 \\
\text { subjectto }
\end{array}}^{\mathrm{K}} \mathrm{a}\left(\widehat{\theta_{\mathrm{k}}}\right) \mathrm{a}^{*}\left(\widehat{\theta_{\mathrm{k}}}\right)
\end{gathered}
$$

In many cases we wish to focus our transmitter energy to one desire direction, tracking applications are such cases. To simplicity and without loss of generality we will consider the case which focusing the total energy at $\theta=0$ is of our interest. In other words it is assume that there is only one target which is placed at $\theta=0$ angle. In such a case our optimization problem will convert to:

$$
\begin{gathered}
\max \operatorname{tr}(\mathrm{R} \widehat{\mathrm{B}}) \\
\mathrm{a}=\text { allonematric } \\
\widehat{\mathrm{B}}=\mathrm{a}(\widehat{\theta}) \mathrm{a}^{*}(\widehat{\theta}) \\
\text { subjectto }|a|=1
\end{gathered}
$$

We will solve this optimization problem with some numerical and iterative method and show the result at the next section. There is one important point at this solution, regardless of other exist algorithm which optimum the placement of the phased array antennas, that place the antennas at a class of specific places of multiple of half wave length, this algorithm has no such a constraint.

\section{NUMERICAL EXAMPLES}

In this section we will show the results by some numerical examples. We suppose that our antenna has 7 elements as it stated before without loss of generality we suppose that our transmitter's elements are placed along the $\mathrm{z}$-axis and the radiation pattern has been measured at distance of $1 \mathrm{Km}$ far from the transmitter along the $\mathrm{x}$-axis and in a range between $-1 \mathrm{Km}$ and $+1 \mathrm{Km}$ along the $\mathrm{z}$-axis. Fig. 3 shows the results of such geometry for conventional phased arrays and our arrangement of antennas placements.

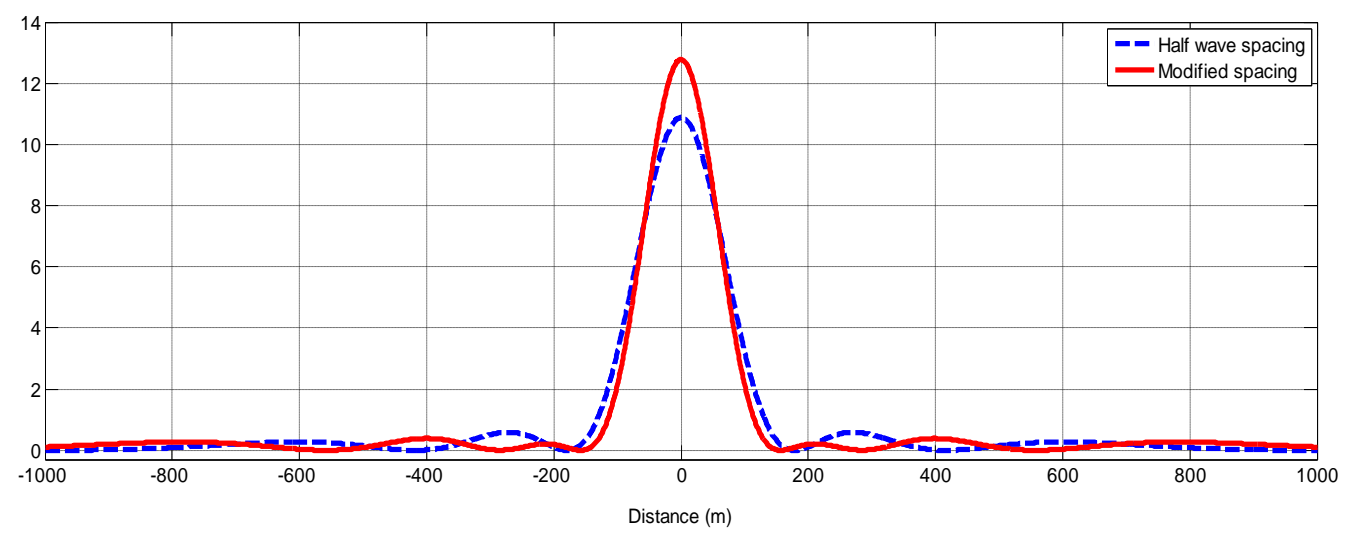

Fig. 3 comparison between uniform and non uniform linear array beampattern

In this figure blue plot is beam-pattern of conventional phased array transmitter that is obtained from equation (7) which has 7 elements in it's transmitter antenna with half wave length spacing between each antennas:

$$
Z=\frac{\lambda}{2}[-3,-2,-1,0,1,2,3]
$$

The red plot in Fig. 3 Shows the beampattern which result from our algorithm that is obtained from two iterations. This result is for below spacing between the antennas elements:

$$
Z=\frac{\lambda}{2}[-3.7,-2.3,-1,0,1,2.2,3.7]
$$

As it seen from this figure with modifying of spacing between elements result from covariance based method we achieve to lower sidelobe level and better transmitter beam-pattern. As we continue the presented algorithm iterations we will achieve more convenient results. To show the usefulness of this algorithm we wish to consider another example. In this example we assume that we have a planar array with $7 * 7$ elements along the $\mathrm{z}$-axis and y-axis. 
Just like the latter example, for conventional phased array we use half wave length spacing between transmitter's elements in both $\mathrm{z}$ and $\mathrm{y}$ axis as shown in Fig. 4 and for modified phase array we use of spacing from presented algorithm like Fig. 5.

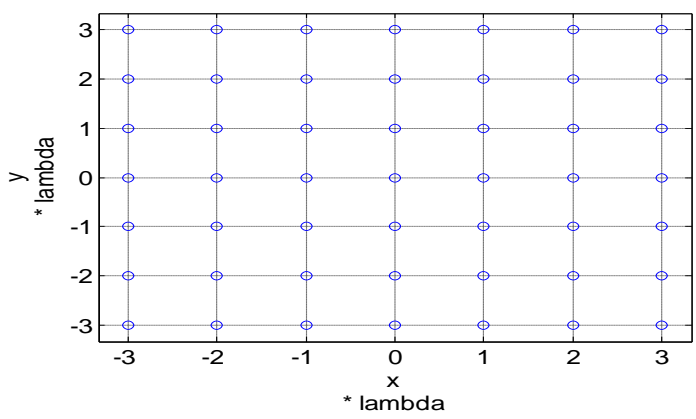

Fig. 4uniform planar array

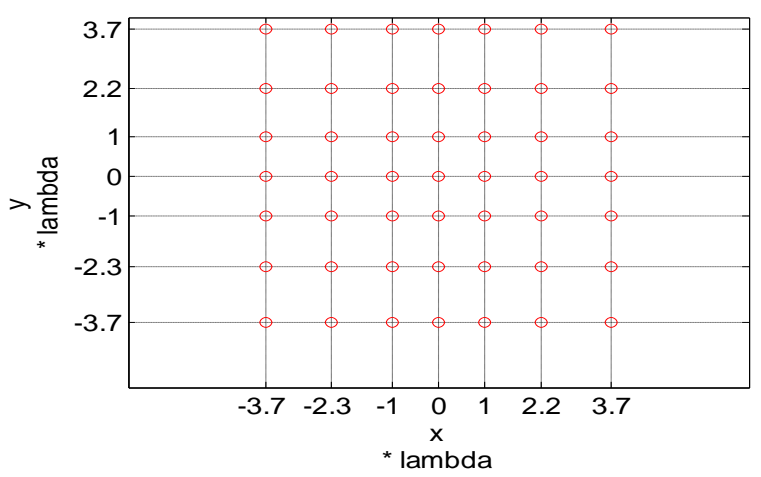

Fig. 5non uniform planar array

Resulting beam-patterns are shown in Fig. 6 and Fig 7. other parameters like distance to measuring the power is the same as latter example. As it is seen from this figure, the resulting beam-pattern from our algorithm with modified antenna placement has lower side-lobe level and sharper peak respect to conventional array with half wave spacing.
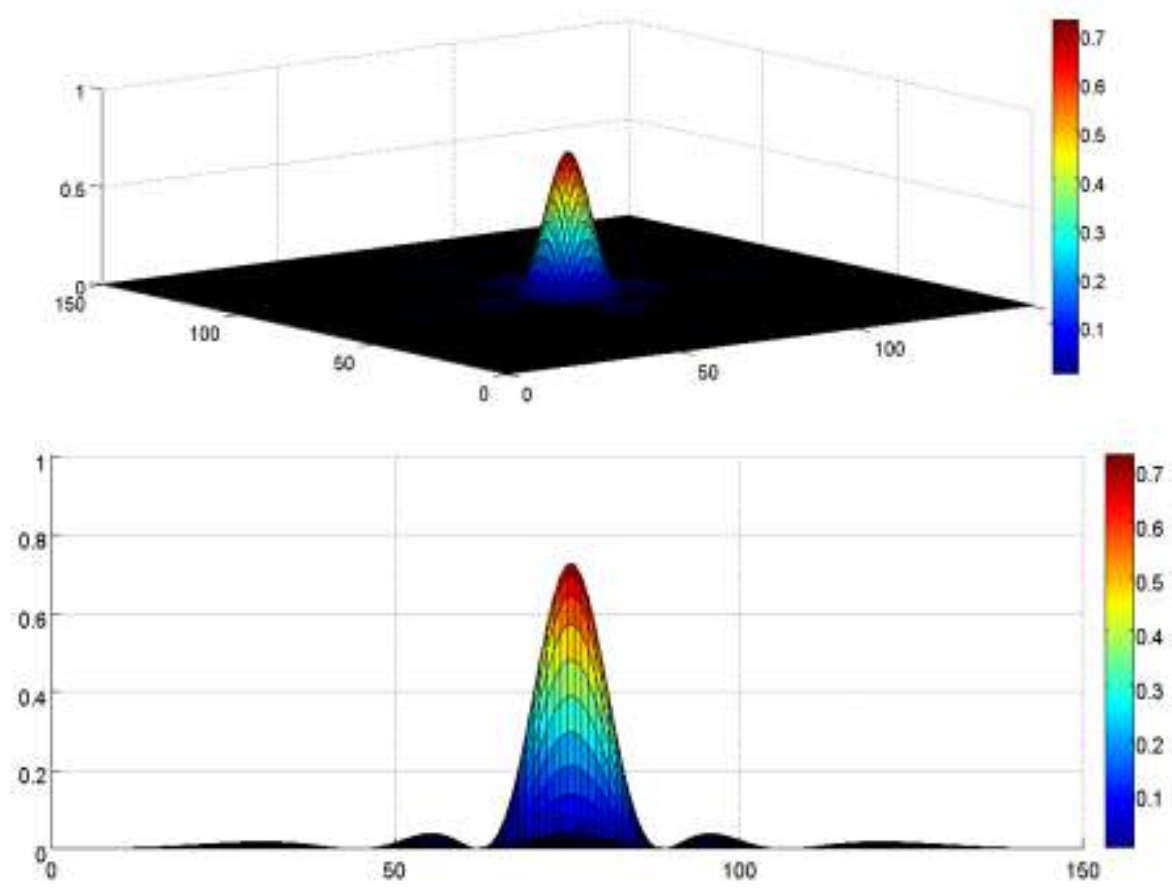

Fig. 6 uniform planar array beampattern 

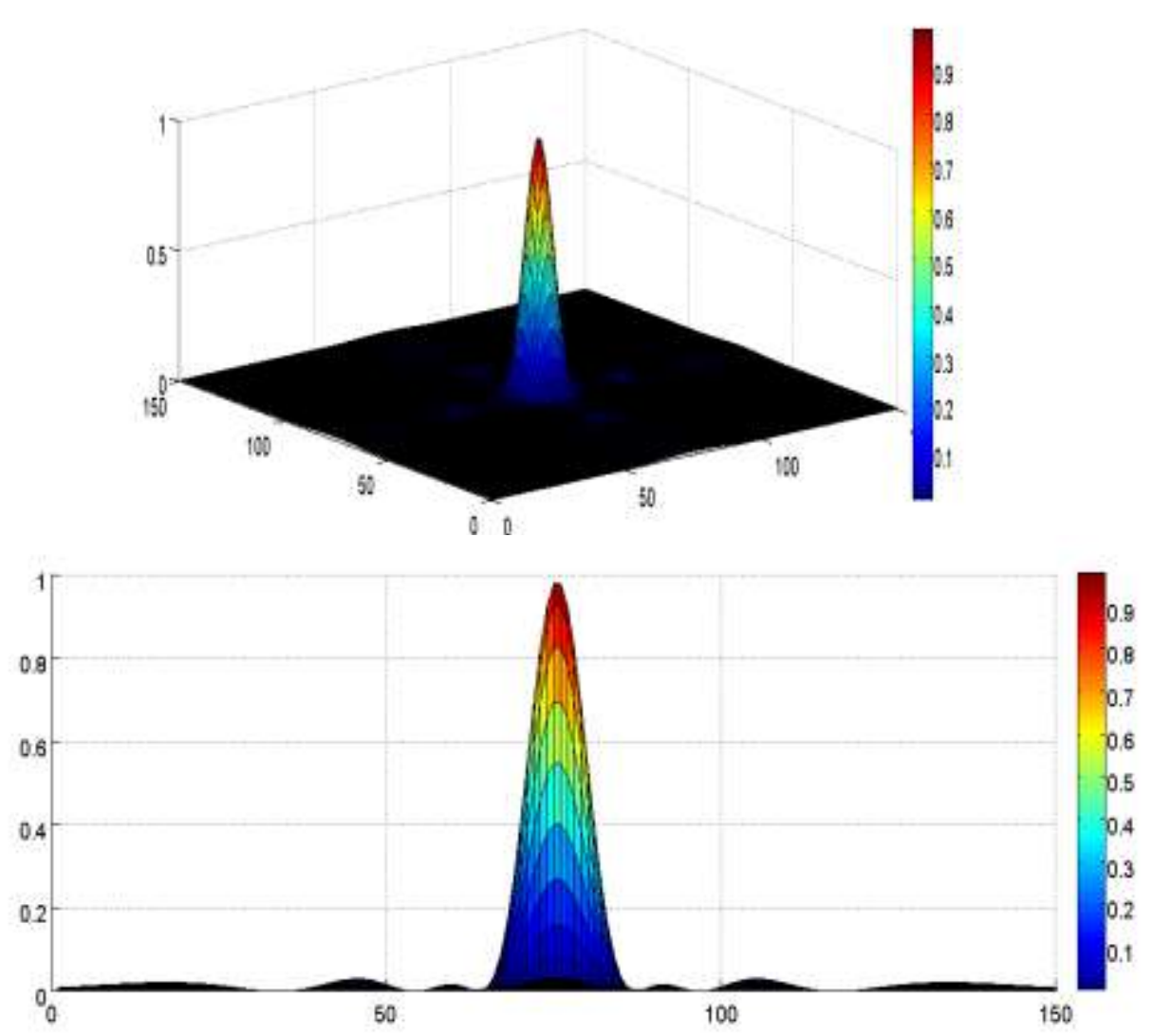

Fig. 7non uniform planar array beampattern

\section{CONCLUSION}

In this paper it presented that with a new approach to defining of beampattern of a phased array antenna based on cross-correlation matrix of transmitter signals, we can have a new vision of optimizing beampattern to steer a desire beampattern energy at a specific direction by optimizing placements of transmitter's antenna elements, also it showed that with this approach we can achieve a pattern that has lower side-lobe level and has sharper peak at desire direction respect to conventional phased arrays with half wave length spacing.

\section{REFERENCES}

[1] M. Stojanovic and Z. Zvonar. "Adaptive Spatial/Temporal Multiuser Receivers for Time-Varying Channels with Severe Isi.” In Proc. of Coq! On Signals and S-ystems, Princeton, March 1994.

[2] B. Ottersten, M. Viberg, P. Stoica, and A. Nehorai. "Exact and large sample ML, techniques for parameter estimation and detection in array processing." In Haykin, Litva, and Shepherd, editors, RadarArray Processing, pages 99-151. Springer-Verlag, Berlin, 1993

[3] S. Shamsunder and G. Giannakis. "Signal Selective Localization of NonGanssian ICyclostationary Sources." IEEE Trans. SP, 42:2860-2864, Oct. 1994.

[4] C.P. Mathews and M. I). Zoltowski. "Eigenstructure Techniques for 2-D Angle Estimation with Unifom Circular Arrays." IEEE Trans. on SP, 42:2395-2407, Sept. 1994

[5] J. Li and P. Stoica. "Efficient Parameter Estimation of Partially Polarized Electromagnetic waves." IEEE Trans. on SP, 42( 11):3 I 14-31 25, Nov. 1994.

[6] H. Krim and J.G. Proakis. "Smoothed Eigenspace-Based Paramerter Estimation." Auromaticu, Special Issue on Statistical Signal Processing and Control, Jan. 1994

[7] J.H. Cozzens and M.J. Sousa. "Source Enumeration in a Conelated Signal Environment." IEEE Trans. on Acoust., Speech and Signal Proc., 42(2):304-317, Feb. 1994.

[8] D. R. Fuhrmann and G. S. Antonio, "Transmit beamforming for MIMO radar systems using partial signal correlation," in Proc. 38th IEEE Asilomar Conf. Signals, Syst., Comput., Nov. 2004, pp. 295-299.

[9] D. R. Fuhrmann and G. S. Antonio, "Transmit beamforming for MIMO radar systems using signal cross-correlation," IEEE Trans. Aerosp. Electron. Syst., vol. 44, pp. 171-186, Jan. 2008.

[10] P. Stoica, J. Li, and Y. Xie, “On probing signal design for MIMO radar,” IEEE Trans. Signal Process., vol. 55, no. 8, pp. 4151-4161, Aug. 2007. 